\title{
Proteins for increased surface expression of the $\alpha 6 \beta 4$ nicotinic acetylcholine receptor: nothing but good news?
}

\author{
Stephen Grant ${ }^{1}$ and Henry A. Lester ${ }^{2}$ \\ 'Division of Chemistry and Chemical Engineering, and 'Division of Biology and Biological Engineering, California Institute of Technology, Pasadena, California, USA.
}

\begin{abstract}
Useful animal models of disease in neuroscience can make accurate predictions about a therapeutic outcome, a feature known as predictive validity. In this issue of the $J C I$, Knowland et al. provide an improved model to assess nicotinic acetylcholine receptor ( $\mathrm{nAChR}$ ) ligands for treating chronic pain. The authors identify two proteins, the voltage-dependent calcium channel auxiliary subunit BARP and the unfolded protein response sensor IRE1 $\alpha$, that are required for robust heterologous expression of $\alpha 6 \beta 4$, an $n A C h R$ subtype in dorsal root ganglia (DRG). This $\mathrm{nAChR}$ is a candidate for the analgesic effects of nicotine as well as the frog toxin epibatidine. Now researchers can efficiently screen for $\alpha 6 \beta 4$ nAChR-selective agonists using heterologous expression systems. Candidates that emerge will enable researchers to test the predictive validity of mouse models for chronic pain in the nAChR context. If all these steps work, one can envision a class of nonopioid nAChR-targeted analgesics for chronic pain.
\end{abstract}

\section{...Nothing but good news \\ There is a frog in South America \\ Whose venom is a cure \\ For all the suffering that mankind \\ Must endure \\ More powerful than morphine \\ And soothing as the rain \\ A frog in South America \\ Has the antidote for pain... \\ - (Paul Simon, "Senorita with a Necklace of Tears")}

\section{The opioid epidemic}

Paul Simon's comparison of epibatidine - the compound secreted by those frogs - with morphine has contemporary relevance. The opioid epidemic continues to ravage, causing approximately one hundred deaths per day in the United States (1). A majority of these opioid overdose deaths come from misused pharmaceutical opioids (2). Unfortunately, 30\% of Americans suffer from some form of pain; for thousands of years, chronic pain has been relieved by opioids. Society continues to struggle to balance several competing issues. (a) Ethical: Some companies encouraged opioid use, a transgression that led to over-prescription. (b) Biological: Opioid use disorder has neurobiological effects ranging from cell biology to behavior. (c) Clinical: Successfully tapering a patient off opioids remains an unsolved problem arising from variations in individual pharmacokinetics and a lack of objective biomarkers for chronic pain. (d) Social: The chemical ease of synthesizing heroin and fentanyl derivatives has enabled people to obtain these opioids to maintain addiction - an issue with inputs from international relations, law enforcement, and education.

A suitable agonist within the theme of $G$ protein-coupled opioid receptors that also allows for reduced dependence represents a long-sought goal $(3,4)$. One pop-

Related Article: p. 6158

Conflict of interest: The authors have declared that no conflict of interest exists.

ular strategy is to develop a biased agonist. Simply stated, a biased opioid receptor agonist activates the appropriate $G$ protein but fails to recruit $\beta$-arrestin, thus reducing tolerance and dependence. Unfortunately, efforts in revealing such an agonist have not yet led to success in the clinic.

\section{The analgesic target for epibatidine has posed challenges}

For several decades, scientists have known that epibatidine acts on most nicotinic acetylcholine receptors (nAChRs), which comprise another signaling pathway for pain relief as well as drugs of abuse (5-7). There are three challenges that limit clinicians from optimally modulating the analgesic properties of nAChRs: (a) Identifying which nAChR constitutes the major analgesic target. (b) Developing a nicotinic agonist that activates $\alpha 4 \beta 2$-containing $\mathrm{nAChRs}$ without leading to the varied and complex dependence pathways downstream from nicotine. (c) Utilizing heterologous expression systems suitable for addressing point (b) with modern drug screening methods.

Seventeen different mammalian subunits $(\alpha 1-\alpha 10, \beta 1-\beta 4, \gamma, \varepsilon$, and $\delta$ ) assemble to form pentameric ligand-gated ion channels that establish an unknown but large number of nAChR subtypes (8). Although many of these receptors are readily expressed in various recombinant expression systems, some require additional proteins to help assembly and trafficking to the plasma membrane. An earlyresearched example is the $\alpha 7 \mathrm{nAChR}$, which generally requires coexpression of chaperone proteins, like Ric3 or NACHO, to conduct meaningful experiments (9, 10). Some small-molecule $\alpha 4 \beta 2$ ligands, such as nicotine, also enhance nAChR surface expression; these ligands act as pharmacological chaperones, binding to nascent receptors in the cytoplasm, and aiding in assembly and trafficking $(11,12)$.

Because the pharmacology of $\alpha 6 \beta 2$ containing and $\alpha 6 \beta 4 \mathrm{nAChRs}$ strongly 
resembles that of $\alpha 4 \beta 2 \mathrm{nAChRs,}$ it has also been suspected that $\alpha 6$-containing nAChRs are also targets for nAChR agonists' analgesic properties (13). The Gordian knot has been achieving functional expression of a6-containing nAChRs. Suboptimal methods to attain functional expression have included engineering mutations that produce hypersensitive receptors (14), that aid trafficking (15), or that enhance expression (16).

\section{Players revealed by cotransfection}

The group at Janssen Pharmaceutical Companies of Johnson \& Johnson has systematically been identifying chaperone proteins for $\mathrm{nAChR}$ expression over the past few years; happily, in this issue of the JCI, they now report a combination of proteins that yield good expression of wild-type $\alpha 6 \beta 4$ nAChRs in recombinant systems (17). The top two proteins that increased the nicotine-evoked $\mathrm{Ca}^{2+}$ response in unbiased genome-wide cotransfection were sodium channel $\beta$-subunit-anchoring and -regulatory protein (BARP) and IRE1 $\alpha$, an unfolded protein response (UPR) sensor. The experiments did not re-identify NACHO, an otherwise promiscuous accessory protein for nAChR expression that increases $\alpha 7, \alpha 4 \beta 2$, $\alpha 3 \beta 2$, and $\alpha 3 \beta 4$ (18).

In Knowland et al.'s experiments, simply expressing $\alpha 6$ and $\beta 4$ (in Xenopus oocyte or HEK293T cell systems) failed to induce ACh-evoked currents, but cells coexpressing BARP generated substantial currents. BARP also increased $\alpha 6 \beta 4$ plasma membrane (PM) expression on HEK293T cells, but failed to induce changes in epibatidine binding. In contrast, coexpression with IRE1 $\alpha$ failed to evoke ACh currents or change PM expression, but increased $\left[{ }^{3} \mathrm{H}\right]$ epibatidine binding (presumably to assembled intracellular nAChRs), suggesting that IRE1 $\alpha$ enhances receptor assembly (17). These results suggest that BARP and IRE1 $\alpha$ affect $\alpha 6 \beta 4$ through distinct mechanisms.

The IRE1 $\alpha$ results are striking because this protein is thought to sense ER stress rather than to enhance protein levels directly. For many membrane proteins, simply improving their folding and assembly within the ER increases PM protein levels (19), as though the rate-limiting step in protein levels at the PM is ER-resident folding and assembly. Indeed, an increase in ER exit sites accompanies both pharmacological chaperoning and increased surface levels $(11,20)$. Although some membrane proteins, for instance some voltage-gated proton channels, spend more time in the Golgi than the ER (21), this is a rare property in the nAChR field. The Knowland et al. results (17) remind us that IRE1 $\alpha$ acts, via its classical XBP1 splicing pathway, during the UPR to reprogram several aspects of ER function. The recent data on IRE1 $\alpha$ present a more general context for the previous suggestion that suppressing the UPR via nicotine-nAChR interactions might benefit people with early-stage Parkinson's disease (22).

The primary motivation to search for these accessory proteins was to help identify the nAChR subunits responsible for the ameliorative pain effects (specifically, antiallodynia) following nAChR stimulation. The dorsal root ganglia (DRG) play a role in nociception and coexpress $\alpha 6$ and $\beta 4$ mRNA (23). Consistent with the experiments performed in HEK293T cells, wild-type DRG neurons had greater $\alpha 6 \beta 4$ surface levels than BARP-KO neurons (17). Nicotine has an antiallodynic effect in rodent models of neuropathic pain (24). Consistent with these previous observations, nicotine decreased mechanical allodynia in the spared nerve injury (SNI) model for wild-type animals, but failed to provide relief to $B A R P-\mathrm{KO}$ samples. To determine whether $\alpha 6 \beta 4$ was the chief target for nicotine's antiallodynic effects, Knowland et al. studied NACHO-KO mice, which would have substantially reduced nAChR expression except for $\alpha 6 \beta 4$. The authors found no difference between nicotine's antiallodynia properties in $\mathrm{NACHO}$ KO mice compared to wild-type mice, consistent with the paper's viewpoint that $\alpha 6 \beta 4$ is the nAChR that carries out nicotine's antiallodynia effects. Inhibition of $\alpha 4 \beta 2$ did not prevent nicotine-induced antiallodynia (17).

\section{Conclusion}

While $\alpha 6 \beta 4$ in DRG neurons plays a key role in nicotine-induced antiallodynia, dopaminergic neurons of the brain express an especially varied collection of other nAChR subtypes. The Janssen Pharmaceutical group recently identified accessory proteins that increase the protein levels of one such subtype, the $\alpha 6 \beta 2 \beta 3 \mathrm{nAChR}$ (25). One therefore hopes that both Parkinson's disease and nicotine dependence may also soon benefit from the ability to screen a full collection of nAChRs (17).

As a caution, we do not yet know of a nicotinic agonist that affects $\alpha 6$-containing AChRs more potently than $\alpha 4$-containing nAChRs. ABT-894, one of the synthetic ligands tested by Knowland et al., has the opposite selectivity, with higher agonist activity at $\alpha 4$-containing nAChRs (17). We can hope that enhanced $\alpha 6 \beta 4$ levels at the plasma membrane will enable experiments to find an $\alpha 6 \beta 4$-selective ligand, thus providing a compound to test the hypothesis that such drugs will become nonaddictive opioids. We must remember that all the indications noted here - antiallodynia, nicotine dependence, and Parkinson's disease - require chronic administration of drugs, which may present additional problems. Overcoming such intellectual and technical barriers would constitute Paul Simon's "Nothing but good news," indeed.

\section{Acknowledgments}

SG and HAL are supported in part by NIH grants DA046122, GM123582, and MH120823.

Address correspondence to: Henry A. Lester, Department of Biology and Biological Engineering 156-29, California Institute of Technology, Pasadena, California 911252900, USA. Phone: 818.422.8169; Email: Lester@Caltech.edu.

1. Kaye AD, et al. New opioid receptor modulators and agonists. Best Pract Res Clin Anaesthesiol. 2018;32(2):125-136.

2. Volkow ND, McLellan AT. Opioid abuse in chronic pain - misconceptions and mitigation strategies. N Engl J Med. 2016;374(13):1253-1263.

3. Kenakin T, Watson C, Muniz-Medina V, Christopoulos A, Novick S. A simple method for quantifying functional selectivity and agonist bias. ACS Chem Neurosci. 2012;3(3):193-203.

4. Gabriel RA, Swisher MW, Sztain JF, Furnish TJ, Ilfeld BM, Said ET. State of the art opioidsparing strategies for post-operative pain in adult surgical patients. Expert Opin Pharmacother. 2019;20(8):949-961.

5. Badio B, Daly JW. Epibatidine, a potent analgetic and nicotinic agonist. Mol Pharmacol. 1994;45(4):563-569.

6. Barreto de Moura F, Withey SL, Bergman J. Enhancement of opioid antinociception by nicotine. JPharmacol Exp Ther. 2019;371(3):624-632.

7. Zhang J, et al. Analgesic effects mediated by neuronal nicotinic acetylcholine receptor ago- 
nists: Correlation with desensitization of $\alpha 4 \beta 2^{*}$ receptors. Eur J Pharm Sci. 2012;47(5):813-823.

8. Gotti C, Zoli M, Clementi F. Brain nicotinic acetylcholine receptors: native subtypes and their relevance. Trends Pharmacol Sci. 2006;27(9):482-491.

9. Treinin M, Jin Y. Cholinergic transmission in C. elegans: Functions, diversity, and maturation of ACh-activated ion channels [published online August 31, 2020]. J Neurochem. https://doi. org/10.1111/jnc.15164.

10. Blunt CEW, Dougherty DA. Binding interactions of NS6740, a silent agonist of the $\alpha 7$ nicotinic acetylcholine receptor. Mol Pharmacol. 2019;96(2):212-218

11. Srinivasan R, et al. Nicotine up-regulates $\alpha 4 \beta 2$ nicotinic receptors and ER exit sites via stoichiometry-dependent chaperoning. J Gen Physiol. 2011;137(1):59-79.

12. Henderson BJ, et al. Menthol stereoisomers exhibit different effects on $\alpha 4 \beta 2 \mathrm{nAChR}$ upregulation and dopamine neuron spontaneous firing. eNeuro. 2019;5(6):ENEURO.0465-18.2018.

13. Wieskopf JS, et al. The nicotinic $\alpha 6$ subunit gene determines variability in chronic pain sensitivity via cross-inhibition of $\mathrm{P} 2 \mathrm{X} 2 / 3$ receptors. Sci
Transl Med. 2015;7(287):287ra72.

14. Drenan RM, et al. In vivo activation of midbrain dopamine neurons via sensitized, high-affinity alpha 6 nicotinic acetylcholine receptors. Neuron. 2008;60(1):123-136.

15. Post MR, Limapichat W, Lester HA, Dougherty DA. Heterologous expression and nonsense suppression provide insights into agonist behavior at $\alpha 6 \beta 2$ nicotinic acetylcholine receptors. Neuropharmacology. 2015;97:376-382.

16. Lowe JA, et al. A novel series of [3.2.1] azabicyclic biaryl ethers as alpha3beta 4 and alpha6/4beta 4 nicotinic receptor agonists. Bioorg Med Chem Lett. 2010;20(16):4749-4752.

17. Knowland D, et al. Functional $\alpha 6 \beta 4$ acetylcholine receptor expression enables pharmacological testing of nicotinic agonists with analgesic properties. JClin Invest. 2020;130(11):6158-6170.

18. Matta JA, et al. NACHO mediates nicotinic acetylcholine receptor function throughout the brain. Cell Rep. 2017;19(4):688-696.

19. Henderson BJ, Lester HA. Inside-out neuropharmacology of nicotinic drugs. Neuropharmacology. 2015;96(pt B):178-193.

20. Henderson BJ, Wall TR, Henley BM, Kim CH, McKinney S, Lester HA. Menthol enhances nicotine reward-related behavior by potentiating nicotine-induced changes in $\mathrm{AAChR}$ function, nAChR upregulation, and DA neuron excitability. Neuropsychopharmacology. 2017;42(12):2285-2291.

21. Ribeiro-Silva L, Arcisio-Miranda M. Molecular basis of voltage-gated proton channel's intracellular trafficking. Biophys J. 2020;118(3). https:// doi.org/10.1016/j.bpj.2019.11.1579.

22. Srinivasan $\mathrm{R}$, et al. Smoking-relevant nicotine concentration attenuates the unfolded protein response in dopaminergic neurons. JNeurosci. 2016;36(1):65-79.

23. Ray P, et al. Comparative transcriptome profiling of the human and mouse dorsal root ganglia: an RNA-seq-based resource for pain and sensory neuroscience research. Pain. 2018;159(7):1325-1345.

24. Kyte SL, et al. Nicotine prevents and reverses paclitaxel-induced mechanical allodynia in a mouse model of CIPN. J Pharmacol Exp Ther. 2018;364(1):110-119.

25. Gu S, Matta JA, Davini WB, Dawe GB, Lord B, Bredt DS. $\alpha 6$-Containing nicotinic acetylcholine receptor reconstitution involves mechanistically distinct accessory components. Cell Rep. 2019;26(4):866-874.e3. 